\title{
Cytidine Triphosphate
}

National Cancer Institute

\section{Source}

National Cancer Institute. Cytidine Triphosphate. NCI Thesaurus. Code C126896.

A cytosine derived nucleotide comprised of three phosphate groups esterified to the deoxyribose sugar moiety. Nucleotide metabolism is upregulated in cancer cells;

therefore, this nucleotide could be used as a biomarker to detect the presence of cancer cells. 